\title{
Nanosilver: a catalyst in enzymatic hydrolysis of starch
}

\author{
Marta Falkowska*, Eugeniusz J. Molga \\ Warsaw University of Technology, Faculty of Chemical and Process Engineering, ul. Waryńskiego 1, 00-645 Warsaw, Poland \\ "Corresponding author: e-mail: M.Falkowska@ichip.pw.edu.pl
}

\begin{abstract}
Silver nanoparticles are widely used, because of their antimicrobial properties. In this paper, the rate of starch digestion in the presence of nanocatalyst was compared with the rate of reaction without nanosilver. The rate of enzymatic degradation of starch was found to be increased in the presence of silver nanoparticles. It is considered that $\alpha$-amylase was immobilized onto the surface of nanoparticles.
\end{abstract}

Keywords: nanocatalyst, starch degradation, nanosilver.

\section{INTRODUCTION}

Green Chemistry is, as the IUPAC definition explains, a design of chemical products and processes that reduce or eliminate the use or generation of substances hazardous to humans, animals, plants and the environment. The implementation of rules of sustainable development and adaptation of 12 fundamental principles of Green Chemistry in every industrial process is crucial. In Figure 1 , there are main concepts of Green Chemistry presented. Catalysis is one of very important principles of Green Chemistry.

The development of catalyst that have high activity and selectivity as well as can be easily removed from reaction medium and recycled, is desirable. Catalysts' higher activity helps to avoid carrying out reactions under drastic conditions (i.e. high temperature, high pressure) and improves the safety of the process. Also an energy efficiency can be improved. The usage of catalysts with higher selectivity by reducing the quantity of by-products formed, reduces costs of main product purification ${ }^{1}$. Obtaining catalysts with these properties became more achievable, because of the application of nanotechnology.

A nanocatalyst is a substance with at least one dimension in nano-scale that shows catalytic influence on chemical reaction process. Acting of nano-sized catalysts is unique, because they bond advantages of homogeneous (high surface area, easily accessible) and heterogeneous (stability, ease of separation and recovery) catalyst system with the limitation of their disadvantages. Morphology, size, shape and composition of nanoparticles have influence on their physical and chemical properties, also on activity and selectivity of nanocatalysts.

The interest of nanocatalysts and their various applications in industry is still increasing. In our previous work $^{2}$ the basic characterization of nanocatalysts and their last applications were collected. One of the widely used and easy to prepare catalyst is nanosilver. Due to its thermal conductivity, chemical stability, catalytic and antibacterial activity, nanosilver can be used not only in catalysis, but also in biotechnology, food, pharmaceutical and cosmetics industry ${ }^{3}$.

In this work nanosilver particles (AgNPs) were obtained and their influence on rate of enzymatic degradation of soluble starch (amylose) by $\alpha$-amylase was investigated. Amylose is a polymer formed by the $\alpha-1,4$ linkages between D-glucose units. Those linkages are cut during the endohydrolysis by $\alpha$-amylase. That enzyme is present in saliva and the animals' pancreas. The products of degradation of amylose are maltose and glucose.

\section{EXPERIMENTAL}

\section{Chemicals and reagents}

All of chemicals and reagents used in experiments were of high purity. $\alpha$-amylase extracted from porcine pancreas (activity $\geq 10$ unit/mg) and 3,5-Dinitrosalicylic acid were obtained from Sigma Aldrich. Soluble starch, silver nitrate, Potassium sodium tartrate and buffer solution 6.86 were obtained from Chempur. In all experiments only ultra-pure water (Mili- Q) was used.

\section{Synthesis of nanosilver}

Nanosilver of uniform size was prepared by the reduction of silver nitrate in the presence of soluble starch, following the method reported by Vigneshwaran et al. ${ }^{4}$. In this synthesis soluble starch acted not only as a reducing agent, but also as a stabilizer for obtained nanoparticles - to prevent them from aggregation. Silver nitrate $(100 \mathrm{mM})$ was added to $1 \%$ soluble starch and mixed for $12 \mathrm{~h}$. Then the solution was autoclaved at $121^{\circ} \mathrm{C}, 103 \mathrm{kPa}$ for $5 \mathrm{~min}$. The presence of nanoparticles was noticed by change of solution's colour. The solution was cooled and used for analysis.

\section{UV-Vis spectral studies}

The presence of synthesized nanosilver particles was investigated by UV-Vis analysis. The spectral studies were carried out on Spectrophotometer Helios Gamma (Thermo Electron Corporation). Samples were tested in the range between 250 and $800 \mathrm{~nm}$.

\section{Nanoparticle Tracking Analysis}

The size of synthesized nanoparticles was investigated by nanoparticles tracking analysis with the use of NanoSight LM10. The mean diameter and the concentration of nanoparticles in a 1000x diluted solution were measured.

\section{Starch degradation}

The soluble starch hydrolysis was carried out under isothermal conditions. Tests were performed in tubes containing starch as a control and starch as a test. Equal amounts of $\alpha$-amylase were added to each tube. The scheme of experiments is shown in Table 1. The sample A cointauned no nanoparticles, the sample B $-1 \mathrm{ml}$ of nanoparticles' solution, the sample $\mathrm{C}-2 \mathrm{ml}$ of nanoparticles' solution. Tubes were incubated at room temperature for $30 \mathrm{~min}$. Samples 
Table 1. Scheme of experiments

\begin{tabular}{|l|c|c|c|c|}
\hline & BLANK & Reaction solution without AgNPs & Reaction solution with 1 ml AgNPs & Reaction solution with 2 ml AgNPs \\
\hline Tube & & A & B & C \\
\hline Soluble starch & $0.5 \mathrm{ml}$ & $0.5 \mathrm{ml}$ & $0.5 \mathrm{ml}$ & $0.5 \mathrm{ml}$ \\
\hline Buffer solution & & $0.5 \mathrm{ml}$ & $0.5 \mathrm{ml}$ & $0.5 \mathrm{ml}$ \\
\hline Water & $1 \mathrm{ml}$ & - & - & - \\
\hline AgNPs solution & - & - & $1 \mathrm{ml}$ & $2 \mathrm{ml}$ \\
\hline$\alpha$-amylase & - & $0.1 \mathrm{ml}$ & $0.1 \mathrm{ml}$ & $0.1 \mathrm{ml}$ \\
\hline \multicolumn{2}{l|}{ Volume of reaction solution } & $1.1 \mathrm{ml}$ & $2.1 \mathrm{ml}$ & $3.1 \mathrm{ml}$ \\
\hline
\end{tabular}

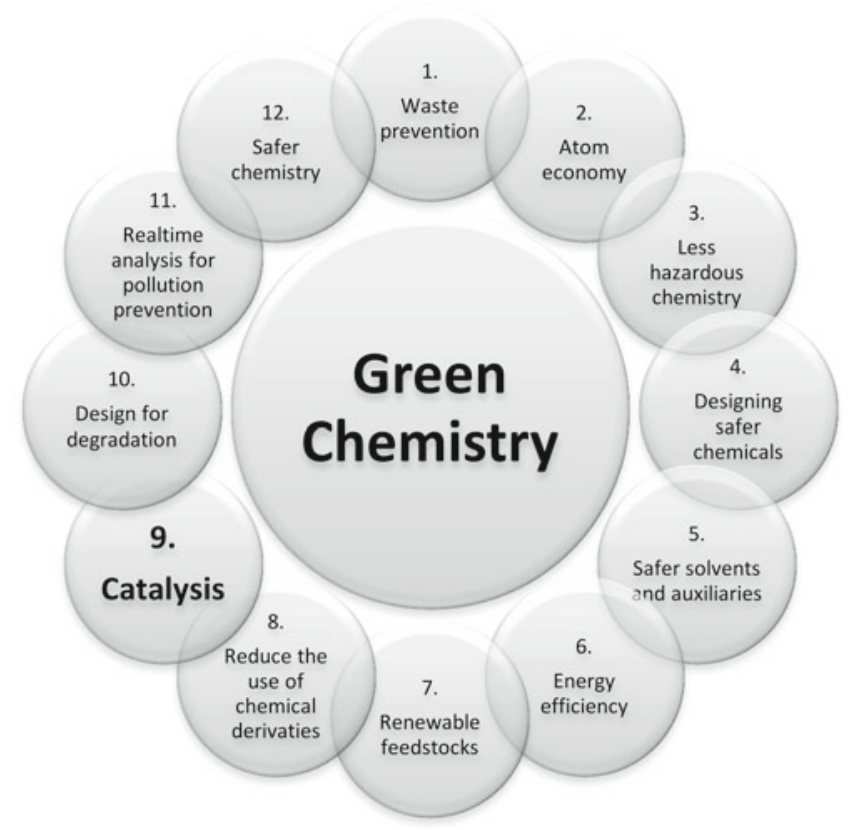

Figure 1., 12 principles of Green Chemistry

were taken at regular intervals and Color Reagent Solution was added to assay the reducing sugar concentration in colorimeter at $540 \mathrm{~nm}$, according to Miller's method ${ }^{5}$. The concentration of reducing sugars was quantified using the maltose standard curve.

\section{RESULTS AND DISCUSSION}

The solution's colour changed from transparent to pale yellow after autoclaving. That indicated the presence of nanoparticles. It was confirmed by UV-Visible spectral studies.

Furthermore, the nanoparticle tracking analysis confirmed presence of nanosilver. The average size of nanosilver was found to be $40 \mathrm{~nm}$. The distribution and the cumulative distribution of nanosilver diameter are shown in Figure 2. The concentration of nanosilver was $11.47 \times 10^{11}$ particles per $\mathrm{ml}$. The method proposed by Vigneshwaran et al. ${ }^{4}$ and further exploited by Ernest et. al. ${ }^{6}$ seems to be a simple and cheap way to produce silver nanoparticles. Nanosilver particles are likely to agglomerate, but the solution with starch synthesized nanosilver was stable for one month.

A relation between products concentration $w_{\mathrm{P}}[\mathrm{mg} / \mathrm{ml}]$ and time of reaction is presented in Figure 3. The products of soluble starch degradation by $\alpha$-amylase are reducing sugars. Their concentration was quantified by Miller's method. Figure 3. shows that the highest concentration of reducing sugars was obtained for the measurement without nanoparticles (A). The volume of reaction solution in that case was the lowest, so the concentration of starch (substrate) was the highest. Due to the variety in the reaction solutions' volume, it is better to compare the temporary rate of production of reducing sugar $R[\mathrm{mg} / \mathrm{min}]$. It was calculated from (1):

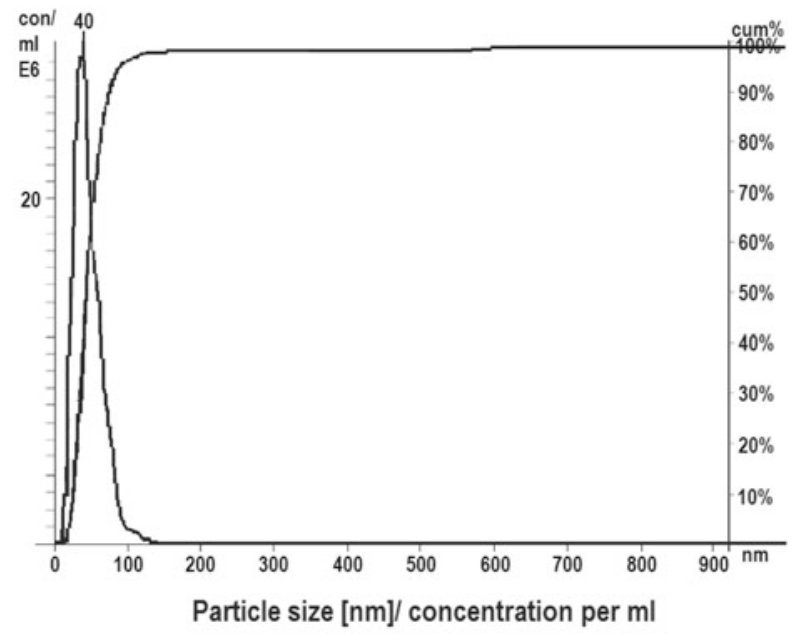

Figure 2. Distribution and cumulative distribution of nanosilver diameter (dilution: 1000x)

$R=\frac{d m_{P}}{d t}=\mathrm{V}_{\mathrm{R}} \frac{\mathrm{dw}_{\mathrm{p}}}{\mathrm{dt}}$

where: $V_{\mathrm{R}}[\mathrm{ml}]$ - volume of the reaction solution, $m_{\mathrm{P}}[\mathrm{mg}]$ - mass of product in the reaction solution, $\mathrm{w}_{\mathrm{P}}[\mathrm{mg} / \mathrm{ml}]-$ concentration of product in the reaction solution.

In Figure 4., the relation between temporary rate of production of reducing sugar $R$ [mg/min] and time is presented. The graph shows the higher temporary rate of production of reducing sugars for measurements with the presence of nanoparticles $(\mathrm{B}, \mathrm{C})$. Furthermore, the concentration of soluble starch in those tubes was lower that in the one without nanoparticles. It proves that the presence of AgNPs increases the rate of enzymatic degradation of starch by $\alpha$-amylase. It is considered that $\alpha$-amylase was immobilized onto the surface of nanoparticles ${ }^{6}$.

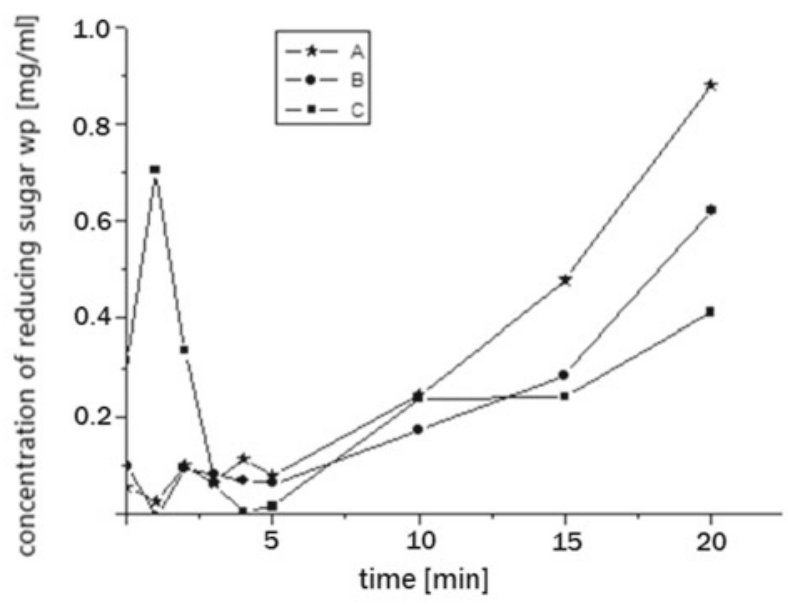

Figure 3. The relation between concentration of reducing sugar and time of reaction 


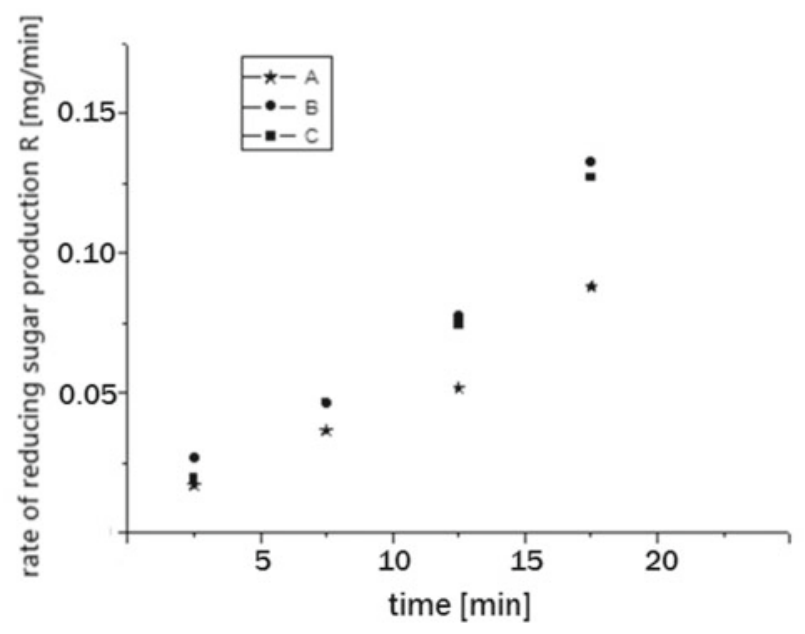

Figure 4. The relation between rate of production of reducing sugar and time of reaction

\section{CONCLUSION}

The aim of present work was to investigate the enzyme-catalysed starch degradation in the presence of nanocatalyst (nanosilver) and to study its kinetics. An influence of nanocatalyst concentration on rate of reaction was studied. It has occurred that the presence of nanosilver accelerates the rate of enzymatic degradation of soluble starch. The novel reaction seems to be more effective method for obtaining reducing sugar than the current one. It could be proposed to industry, but also the toxicity of nanosilver and its recovery scheme should be considered.

Our future goal is to investigate the mechanism of soluble starch enzymatic degradation with usage of nanosilver acting as a catalyst.

Furthermore, the method used for synthesis of nanosilver was found to be very stable, easy and not requiring high costs.

\section{ACKNOWLEDGMENT}

Authors are thankful to Prof. Tomasz Ciach (Faculty of Chemical and Process Engineering, Warsaw University of Technology) for providing specialized measuring equipment and Msc. Eng. Iga Wasiak and Msc. Eng. Magdalena Janczewska for their help with measurements.

\section{LITERATURE CITED}

1. Kalidindi, S.B. \& Jagirdar, B.R. (2012). Nanocatalysis and prospect of green chemistry. ChemSusChem. 5, 65-75. DOI: 10.1002/cssc.201100377.

2. Molga, E. \& Falkowska, M. (2012). Nanocatalysts characterization and applications (In Polish). III Polish Symposium „Multiphase and Multifunctional Reactors for Chemical and Environmental Processes", 10-12 October 2012 (pp. 137-142). Warsaw-Serock, Poland, ISBN 978-83-906658-9-4.

3. Cong, H. \& Porco, A.J.Jr. (2012). Chemical Synthesis of Complex Molecules Using Nanoparticle Catalysis. ACS Catalysis. 2, 65-70. DOI: 10.1021/cs200495s.

4. Vigneshwaran, N., Nachane, R.P., Balasubramanaya, R.H. \& Varadarajan, P.V. (2006). A novel one-pot 'gre- en' synthesis of stable silver nanoparticles using soluble starch. Carbohydr. Res. 341, 2012-2018. DOI: 10.1016/j. carres.2006.04.042.

5. Miller, G.L. (1956). Use of dinitrosalicylic acid reagent for determination of reducing sugar. Anal. Chem. 31(3), 426-428. DOI: 10.1021/ac60147a030.

6. Ernest, V., Shiny, P.J., Mukherjee, A. \& Chandrasekaran, N. (2013). Silver nanoparticles: a potential nanocatalyst for the rapid degradation of starch hydrolysis by a-amylase. Carbohydr. Res. 352, 60-64. DOI: 10.1016/j. carres.2012.02.009. 